\title{
Online dental trauma course created to support clinicians
}

Greater Manchester Health \& Social Care Partnership, Local Dental Network and Health Education England have teamed up with the Manchester University NHS Foundation Trust, FDS Referrals and Barts NHS Trust to create an online dental trauma course. The course consists of 13 webinars covering all major topics relevant to dental trauma from diagnosis to management strategies. All of these can be accessed remotely and each module can be dipped in and out of to allow flexible learning. The course is free to access for all and attracts six hours of CPD. Go to: https:// www.bmh.manchester.ac.uk/dental-trauma/ to sign up.
Supporting this is the Saving Smiles Toolkit which can be downloaded and kept in surgeries to support clinicians when they need it the most. Copies can be found at: https://www.dental-referrals.org/wp-content/ uploads/2017/08/Saving-Smiles-V8.pdf.

Contact James Darcey at James.darcey@ mft.nhs.uk for more information.

\section{Team adapt elements of the Spitfire to design new PPE for dentistry}

A multidisciplinary team including an aerodynamicist and aircraft designer, furloughed by the lockdown, volunteered to investigate aerosols, in a bid to designing personal protective equipment (PPE) that is effective, efficient, ergonomic and compliant with the 2020 COVID-19 laws.

Aiming to 'unlock' dentistry and open practices so that patients can be treated, lifting the burden on hospitals, J. Laszlo, R. John, L. Williams, P. Handley and D. Stevenson wrote to the $B D J$ in early May, outlining their investigation.

They write:

'The Office for Product Safety and Standards (OPSS) upholds the requirements governing the design and manufacture of clinical PPE in the UK. In the advent of COVID-19, from April 2020, a legislative easement facilitated the design and release to service of PPE, but such designs must still follow EU Regulation 2016/425 and the Personal Protective Equipment (Enforcement) Regulations 2018 (SI 2018 No. 390). ${ }^{1}$

'An aerosol's turbulence can be controlled by even the smallest of pressure changes, but such effects are best achieved in a small space, by laminar air flow and when the total number of confining flat surfaces, edges and sharp corners are reduced, so stagnation zones in which viral particles concentrate being difficult to disinfect, are eliminated from the design.

'This design is large enough to enclose a patient's head and neck but small enough to be carried, easily disinfected and securely placed onto any one of the many types of dental chair presently in service, in either the upright or the supine position. ${ }^{2}$ A lower than ambient pressure is achieved within a profiled canopy with low-volume dental suction and this reduces, if not entirely removes the risk of external aerosol contamination in the clinic. Access ports can be opened and closed as needed (Fig. 1).

'It was intended that a multidisciplinary team was brought together (albeit virtually) from across the UK, but it was serendipitous that a firm still producing components for the Spitfire, (White-Ellerton Ltd) would open their doors freely providing expertise and materials. Indeed, the project adopted many of Reginald Mitchell's design strategies. It was Neil Williams who said the Spitfire has no straight lines. ${ }^{3}$ The purpose of the product can be seen by the presence of such patterns.

'Mandatory registration with a notifying body for conformity assessment, trade marking and registration has begun (Fig. 2). This allows the design to be released to service

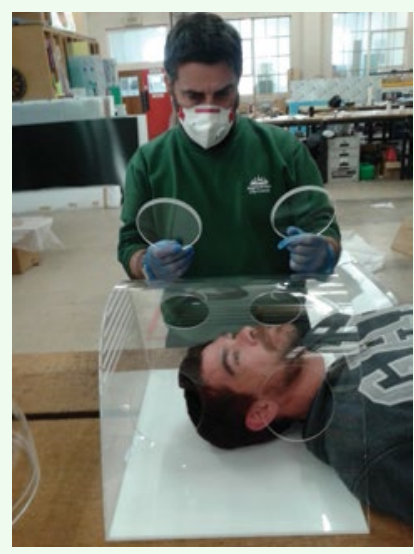

Fig.1 COVID-CANOPY. Canopy ports removed for access to patient. The access panels, optical clarity and the calculated profile curve can be seen in the design that takes much from the patterns of Reginald Mitchell. The canopy is manufactured from CAA approved aviation grade Perspex. A flexible base adapts to dental chairs and is easy to disinfect across the UK in accordance with the 2020 OPSS easement regulations. With reflection, it is humbling to realise 80 years after the Spitfire flew in battle, elements of Mitchell's masterpiece may now play their part unlocking dentistry, defending the UK against a merciless enemy that has taken so many, so quickly. This project is voluntary and dedicated to the front liners who have made the supreme sacrifice.'

Since originally contacting the $B D J$, the team's PPE projects are ready for the next stage of conformity assessments for CE approval (and CE marking) so that they can be delivered to dentists and front line workers as soon as possible.

\section{References}

1. Office for Product Safety \& Standards Personal Protective Equipment (PPE) Regulations OPSS PPE. Legislation Guidance for Businesses, Version 2. April 2020.

2. Mr Paul Smith, Tridac UK personal communication 3 March 2020.

3. Neil Williams (1934-1977), RAF, Spitfire and test pilot, personal communication to Lynn Williams.

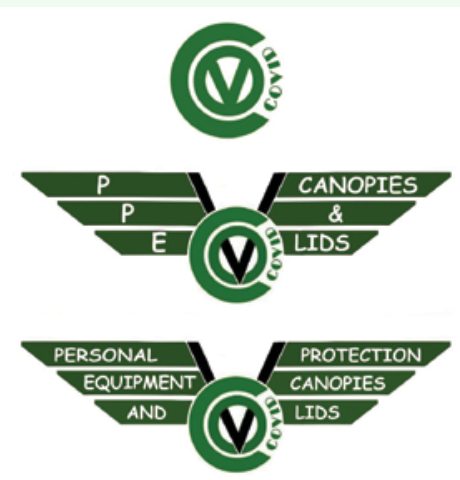

Fig. 2 Project Trademarks Registration Number: UK00003486612. The winged $V$ is for Victory over the C for COVID-19. The affixing of a trademark is a legal requirement as the product can be released to service while awaiting CE mark 